\title{
Modelo de Classificação de Grupos baseado em Rotulação e Lógica Fuzzy
}

\author{
Sidiney S. Araujo* Vinicius P. Machado* \\ Lúcia Emília S. Silva* Rodrigo Veras* \\ * Departamento de Ciência da Computação, Universidade Federal do \\ Piauí,PI, (e-mails: sidiney01@gmail.com,vinicius@ufpi.edu.br, \\ luciaemilia@ufpi.edu.br rveras@ufpi.edu.br).
}

\begin{abstract}
The techniques of clustering and classification are frequently used to obtain patterns and classify new data. The combination of those techniques can be applied to problems where there is no label, using the clustering process to extract information that will assist the classification process. Usually, the clusters are analyzed by an expert to obtain that information, but this process can also be done by automatic labeling models. Those are models capable of identifying the most relevant characteristics and use them to create a label. This paper proposes a model of classification of clusters using labels and Fuzzy logic. The efficiency of the proposed model was evaluated by comparing the accuracy and standard deviation, as well as individually analyzed each rule through the metrics based on the contingency matrix. For different databases available in the UCI repository, the results show that the rules reached precision and specificity rates above $94 \%$, with accuracy and standard deviation similar to the algorithms.

Resumo: As técnicas de agrupamento e classificação de dados são frequentemente utilizadas com a finalidade de extrair padrões e classificar novos elementos. A combinação de tais técnicas pode ser aplicada em bases que não se conhece o atributo classe, utilizando a interpretação dos grupos obtidos no processo de agrupamento para identificação de padrões que auxilie o processo de classificacão. Esta interpretação pode ser desempenhada por modelos de rotulação: modelos capazes de identificar características relevantes e utilizá-las na formação de rótulos. Este trabalho propõe um modelo de classificação de grupos utilizando os rótulos e lógica Fuzzy. O modelo proposto foi avaliado comparando a acurácia e desvio padrão, bem como analisado individualmente cada regra através da matriz de contingência. Para diferentes bases disponíveis no repositório UCI, os resultados mostram que as regras obtidas alcançaram taxas de precisão e especificidade acima de $94 \%$, com acurácia e desvio padrão similares aos de algoritmos comparados.
\end{abstract}

Keywords: Machine Learning; Classification; Fuzzy; Rules; Labeling

Palavras-chaves: Aprendizagem de Máquina; Classificação; Fuzzy; Regras; Rotulação

\section{INTRODUÇÃO}

Com o avanço da informatização, grandes bases de dados são geradas e atualizadas a todo momento possibilitando a aplicação de técnicas para extração de conhecimento, um dos principais objetivos da Aprendizagem de Máquina (AM). Na realização de suas tarefas, AM comumente dividi-se em dois tipos: aprendizagem supervisionada, na qual as classes dos dados são conhecidas; e não supervisionada, na qual a classe dos dados é desconhecida ou omitida. De modo que a aprendizagem supervisionada desempenha tarefas cujo objetivo é a classificação ou regressão de novos dados, enquanto a não supervisionada tem como principal tarefa o agrupamento, em que busca-se formar subconjuntos com dados que possuem características similares entre si e dissimilares com outros subconjuntos (Russell and Norvig, 2016).

\footnotetext{
^ Os autores agradecem a Coordenação de Aperfeiçoamento de Pessoal de Nível Superior (CAPES) por patrocinar nossa pesquisa.
}

Ao longos dos anos surgiram diversas técnicas utilizando AM: na aprendizagem supervisionada por exemplo, algoritmos baseados em Redes Neurais (Silva et al., 2010), Árvores de Decisão (Safavian and Landgrebe, 1991) ou Regras (Nomura et al., 1992) são aplicados em problemas de diversos domínios. Algumas técnicas buscam maiores acurácia nos resultados, como Multilayer Perceptron (MLP) (Silva et al., 2010), outras procuram mostrar o conhecimento obtido de forma a facilitar a interpretação dos resultados como as Árvores de Decisão (Milaré et al., 2002).

A grande variedade de técnicas também está presente na aprendizagem não supervisionada, a exemplo os algoritmos K-Means (MacQueen et al., 1967), Fuzzy C-Means (Bezdek, 1981), CURE (Guha et al., 1998), DBSCAN (Ester et al., 1996), entre outros. O objetivo pincipal desses algoritmos é a formação de grupos através da identificação de similaridades e dissimilares dos dados. 
Um problema relacionado ao agrupamento é a interpretação dos grupos formados que pode ser dificultada pela quantidade de instâncias e atributos presentes na base. Para resolução deste problema tem-se os modelos de rotulação que buscam gerar rótulos que identifiquem de forma única cada grupo a fim de facilitar sua interpretação. Os modelos de rotulação, de modo geral, dão-se pela combinação de técnicas de AM já existentes.

Outro probelma relacionado ao agrupamento é a associação de novos elementos aos grupos formados. Este cenário torna propício a utilização de técnicas baseadas em aprendizagem supervisionada, em que os dados já agrupados são utilizados no treinamento do algoritmo. Apesar de eficiente, o uso de técnicas de classificação em agrupamentos pode não ser sastifatório uma vez que não se conhece quais similaridades formaram os grupos, retornando ao problema de interpretação. Neste sentido, os modelos de rotulação podem ser utilizados como auxílio para análise do agrupamento, fornecendo informações úteis para o treinamento de um modelo de classificação.

Este trabalho propõe um modelo para classificação baseado em lógica Fuzzy com o objetivo de classificar novos elementos através das informações obtidas pela rotulação de grupos.

O restante do trabalho está organizado da seguinte forma: Na Seção 2, são introduzidos conceitos sobre rotulação de grupos, Sistemas Fuzzy e trabalhos relacionados; O modelo proposto, assim como as as bases de dados utilizadas e as métricas de avaliação são apresentados na Seção 3; na Seção 4 são apresentados os resultados dos testes realizados, seguido de uma discussão sobre os mesmos na Seção 5. Por fim, na Seção 6 é exposta a conclusão deste trabalho.

\section{REFERENCIAL TEÓRICO}

\subsection{Rotulação de Grupos}

As técnicas de aprendizagem não supervisionada propõemse a formar grupos de acordo com as medidas de similaridades ou dissimilaridades dos dados (Atilgan and Nasibov, 2016), de modo que a interpretação dos mesmos fica à critério de um especialista. Entretanto, a medida que aumentam os dados, maior a dificuldade de interpretação destes grupos. Neste contexto, a rotulação automática apresenta-se como uma técnica capaz de provêr auxílio ao especialista. Lopes et al. (2016) define que o problema da rotulação consiste em apresentar uma identificação resumida para os grupos, nomeando-os de acordo com suas características. A definição formal do problema de rotulação é apresentada a seguir:

Problema de Rotulação: Dado um conjunto de grupos $C=\left\{c_{1}, \ldots, c_{K} \mid K>1\right\}$, de modo que cada grupo contém um conjunto de elementos $\left\{c_{i}=\boldsymbol{e}_{1}, \ldots, \boldsymbol{e}_{n^{\left(c_{i}\right)}} \mid n^{\left(c_{i}\right)} \geq 1\right\}$ que podem ser representados por um vetor de atributos definidos em $R^{m}$ e expresso por $\boldsymbol{e}_{j}^{\left(c_{i}\right)}=\left(a_{1}, \ldots, a_{m}\right)$, e que $c_{i} \cap c_{i^{\prime}}=\{\emptyset\}$ com $1 \leq i, i^{\prime} \leq K$ e $i \neq i^{\prime} ;$ o objetivo consiste em apresentar um conjunto de rótulos $R=\left\{r_{c_{1}}, \ldots, r_{c_{K}}\right\}$ no qual cada rótulo específico é dado por um conjunto de pares de valores: atributo e seu respectivo intervalo, $\left.\left.r_{c_{i}}=\left\{\left(a_{i},\left[p_{i}, q_{i}\right]\right), \ldots,\left(a_{m^{\left(c_{i}\right)}},\right] p_{m^{\left(c_{i}\right)}}, q_{m^{\left(c_{i}\right)}}\right]\right)\right\}$ tal que $r_{c_{i}}-$ $r_{c_{i^{\prime}}} \neq \emptyset$ e $r_{c_{i}} \not \supset r_{c_{i^{\prime}}}$, seja capaz de melhor expressar o grupo $c_{i}$ associado.

A fim de esclarecimento, tem-se que:

- $K$ é o número de grupos;

- $c_{i}$ é um grupo qualquer;

- $n^{\left(c_{i}\right)}$ é o número de elementos do grupo $c_{i}$;

- $\boldsymbol{e}_{j}^{\left(c_{i}\right)}$ se refere ao j-ésimo elemento pertencente ao grupo $c_{i}$

- $m$ é a dimensão do problema;

- $\left.] p_{m^{\left(c_{i}\right)}}, q_{m^{\left(c_{i}\right)}}\right]$ representa o intervalo de valores $a_{m^{\left(c_{i}\right)}}$ onde $p_{m^{\left(c_{i}\right)}}$ é o limite inferior e $q_{m^{\left(c_{i}\right)}}$ é o limite superior;

- $m^{\left(c_{i}\right)}$ é a quantidade de atributos presente em um rótulo referente ao grupo $c_{i}$.

Diferentes modelos para resolução do problema da rotulação são propostos na literatura. Por exemplo, Lopes et al. (2016) utiliza Redes Neurais Artificiais, enquanto Machado et al. (2015) utiliza a matriz de pertinência resultante do algoritmo Fuzzy $C$-Means.

\subsection{Sistemas Fuzzy}

Sistemas Fuzzy são sistemas baseados nas teorias de conjuntos Fuzzy e lógica Fuzzy, para construção de um raciocínio aproximado, permitindo a expressão da ambiguidade e subjetividade presentes no raciocínio humano (Pedrycz and Gomide, 1998).

A teoria dos conjuntos Fuzzy é considerada uma extensão da teoria clássica de conjuntos (Zimmermann, 1996), na qual um elemento pode apenas pertencer ou não à um conjunto ou classe. O cálculo da pertinência de um elemento em um conjunto Fuzzy é feito através das funções de pertinências, representadas por qualquer função que produza valores no intervalo $[0,1]$, em que 0 representa nenhuma pertinência e 1 total pertinência ao conjunto (Zadeh et al., 1965).

Outra definição utilizada nos conjuntos Fuzzy refere-se as variáveis linguísticas, que segundo Zimmermann (1996) são aquelas cujos valores são palavras ou sentenças em linguagem natural em vez de números. Por exemplo, a variável linguística temperatura pode ter seu domínio granularizado nos valores linguísticos baixa, média e alta.

Os pares variáveis linguísticas e valor linguístico são utilizados como termos para modelagem dos sistemas Fuzzy através de regras Fuzzy da forma:

\section{Se antecedente então consequente.}

Segundo Jamshidi et al. (1993), os Sistemas Fuzzy possuem três etapas: Fuzzyficação, Processo de Inferência e Defuzzyficação. Na Fuzzyficação é realizado o mapeamento do domínio de entrada dos dados reais para o domínio Fuzzy. O Processo de Inferência avalia o conjunto de regras que descrevem a relação entre as variáveis de entrada e saída, ou antecedente e consequente. Em algumas arquiteturas, após o processo inferência é necessário a transformação do valor Fuzzy para uma saída numérica, essa etapa é conhecida como Defuzzyficação. 


\subsection{Trabalhos Relacionados}

Nos últimos anos diversos trabalhos foram publicados apresentando abordagens que combinam técnicas baseadas em aprendizagem não supervisionada e supervisionada. No trabalho de Elyasigomari et al. (2015) é proposta uma abordagem para classificação de tipos de câncer baseada em agrupamentos e otimização. Neste trabalho é mostrado que a classificação de tipos de câncer requer análise de um número extensivo de genes, uma tarefa extremamente complexa se realizada manualmente. A solução apresentada é a seleção de genes realizada por um algoritmo de agrupamento, utilizando uma junção do algoritmo de otimização Cuckoo (COA) e algoritmo genético. Após o agrupamento são selecionados os 20 melhores genes de cada grupo para serem utilizados pelas técnicas de classificação MLP e Support Vector Machine (SVM).

Biscarri et al. (2017) apresentam um framework que utiliza agrupamentos para auxiliar na classificação automática do consumo de energia. Neste trabalho é realizado uma seleção dos dados similares dos consumidores com base no seus consumos e outros critérios de economia, submetendo-os à diferentes algoritmos de agrupamento, como algoritmos de agrupamento hieráquico, $K$-means, Diana, PAM, Clara, Fanny, SOM, algoritmos baseados em modelos e SOTA. A validação do agrupamento é feita pelo especialista da companhia de energia e então submetida para classificação.

Já Gan et al. (2018) propõem uma forma de incrementar a performance de classificação para os métodos baseados de aprendizagem em grafos. O trabalho introduz o algoritmo chamando Classificação de Quadrados Mínimos Regularizados baseado em Agrupamentos Supervisionados (SuperRLSC). O modelo inicialmente aplica um algoritmo $K$-means supervisionado para descobrir estruturas presentes nos dados. Após esta descoberta, as estruturas são utilizadas para criação dos grafos e, por fim, aplica-se um termo de regularização baseados em grafos (RLSC) para classificação. Os resultados obtidos são comparados com outros algoritmos de classificação presentes no estado da arte como RLSC, KMSE, SVM, MR, DRGV e DRLSC.

As abordagens citadas apresentam estruturas similares, consistindo, de modo geral, em: pré-processamento, agrupamento, análise dos grupos e classificação. Dentre as etapas detacamos a análise dos grupos, ora desempenhada por modelos matetáticos, ora por especialistas, despendendo de recursos e tempo em ambos os casos. Neste sentido, no modelo proposto neste trabalho a análise dos grupos é desempenhada de forma direta na geração do classificador.

\section{MATERIAIS E MÉTODOS}

\subsection{Modelo Proposto}

Este trabalho propõe um modelo de classificação baseado em lógica Fuzzy que utiliza os rótulos gerados pelos modelos de rotulação automáticos para formação das regras e funções de pertinência. O modelo está divido em três etapas: formação dos grupos (I), rotulação (II) e geração do classificador (III).

Uma base de dados fictícia, denominada Base de Dados Modelo (BDM), apresentada na Tabela 1, é utilizada para demonstrar o funcionamento do modelo. A base é composta por 30 elementos, representados por um identificador e dois atributos: $A t t r_{1}$ e $A t t r_{2}$.

Tabela 1. Base de Dados Modelo (BDM).

\begin{tabular}{|c|cc|}
\hline$\#$ & Attr $_{1}$ & Attr $_{2}$ \\
\hline 1 & 4,3 & 6,0 \\
\hline 2 & 9,7 & 6,5 \\
\hline 3 & 4,7 & 5,7 \\
\hline 4 & 7,0 & 8,0 \\
\hline 5 & 4,3 & 4,7 \\
\hline 6 & 4,9 & 4,8 \\
\hline 7 & 3,1 & 5,7 \\
\hline 8 & 4,1 & 5,5 \\
\hline 9 & 5,8 & 7,5 \\
\hline 10 & 9,4 & 6,0 \\
\hline 11 & 9,8 & 3,5 \\
\hline 12 & 8,0 & 4,0 \\
\hline 13 & 9,0 & 6,7 \\
\hline 14 & 4,5 & 3,7 \\
\hline 15 & 8,8 & 4,5 \\
\hline
\end{tabular}

\begin{tabular}{|c|cc|}
\hline$\#$ & Atrr $_{1}$ & Attr $_{2}$ \\
\hline 16 & 8,9 & 4,0 \\
\hline 17 & 7,8 & 7,7 \\
\hline 18 & 7,8 & 4,6 \\
\hline 19 & 3,9 & 3,6 \\
\hline 20 & 8,5 & 5,1 \\
\hline 21 & 7,6 & 6,9 \\
\hline 22 & 5,9 & 8,5 \\
\hline 23 & 9,1 & 6,4 \\
\hline 24 & 7,0 & 7,3 \\
\hline 25 & 6,3 & 7,4 \\
\hline 26 & 5,3 & 3,8 \\
\hline 27 & 7,5 & 8,2 \\
\hline 28 & 5,9 & 6,9 \\
\hline 29 & 3,8 & 4,6 \\
\hline 30 & 6,8 & 7,9 \\
\hline
\end{tabular}

Formação dos Grupos Nesta etapa é realizado o agrupamento dos dados. Para exemplificar, é aplicado na BDM o algoritmo $K$-means, com $k$ igual à 3 . Com o resultado obtido pelo K-means é adcionado uma coluna que indica o grupo à que cada elemento foi associado.

Rotulação dos Grupos Para rotulação o modelo proposto por Lopes et al. (2016) é aplicado nos grupos criados. O objetivo é encontrar os pares de atributos e intervalos de valores que melhor representem cada grupo para composição do rótulo.

O modelo de rotulação seleciona os atributos mais relevantes de cada grupo através de Redes Neurais, que para o exemplo da BDM são: Grupo $1=\left\{A t t r_{1}\right\}$, Grupo $2=\left\{A t r_{1}\right\}$ e Grupo $3=\left\{A t r_{1}, A t t r_{2}\right\}$. Em seguida, seleciona-se o valor mais frequente de cada atributo, ou, caso seja um atributo discretizado, o intervalo de valores mais frequente associado à cada atributo. Para nosso exemplo, os rótulos obtidos são apresentados na Tabela 2.

Tabela 2. Rótulos para BDM.

\begin{tabular}{|c|c|c|c|}
\hline & Grupo 1 & Grupo 2 & Grupo 3 \\
\hline Attr $_{1}$ & {$[3,1 \sim 5,3]$} & {$[5.8 \sim 7,8]$} & {$[8,5 \sim 9,8]$} \\
\hline Attr $_{2}$ & - & - & {$[7,3 \sim 8,5]$} \\
\hline
\end{tabular}

Como pode ser observado na Tabela 2, o Attr 1 apresenta faixas de valores que caracterizam cada um dos três grupos, sendo o Grupo 3 representado pela combinação de faixas de valores dos atributlos $A t t r_{1}$ e $A t t r_{2}$. Percebese que quanto maior o número de atibutos no rótulo de um grupo mais específico este é, visto que, para que um elemento pertença ao grupo é necessário que este satisfaça um maior número de restrições.

Geração das Regras e Funções de Pertinência Na etapa III, são geradas as regras e funções de pertinência do modelo proposto utilizando a lógica Fuzzy, com os atributos e intervalos dos rótulos sendo utilizados para cálculo das funções de pertinência.

Para cada atributo presente nos rótulos uma função de pertinência é formada, utilizando cada intervalo de valores para compor uma partição Fuzzy, como por exemplo, para 
a BDM o atributo Attr $_{1}$ está presente nos rótulos dos três grupos, gerando três partições como observado na Figura 1 .

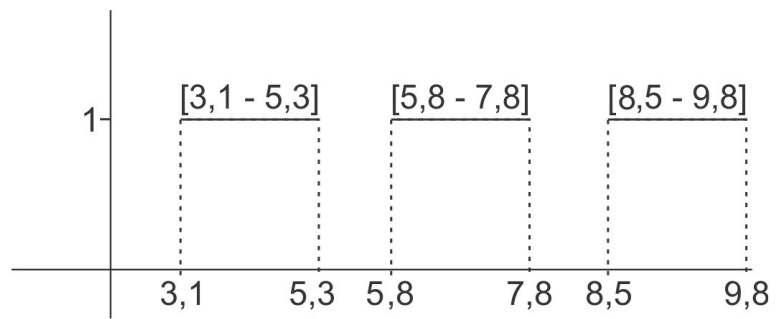

Figura 1. Intervalos do $A t t_{1}$ em que a pertinência aos grupos é 1 .

Observa-se na Figura 1 que existem espaços vazios entre os intervalos tornando inviável a atribuição de um grupo para um elemento que assuma valor nestes espaços. Para isso, segmentos de retas que integrem todos os valores possíveis do atributo às partições são adicionados formando funções da forma trapezoidal.

Dessa forma, o atributo $A t t r_{1}$ da BDM compreende valores entre 3,1 e 9,8. A partir desses valores são calculados os segmentos de retas à serem agregados aos intervalos da Figura 1, como mostrado na Figura 2.

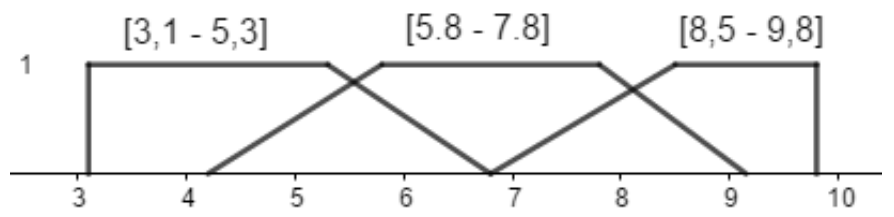

Figura 2. Função de pertinência final do atributo $A t t r_{1}$.

$\mathrm{Na}$ etapa de inferência do Sistema Fuzzy, as regras são formadas a partir dos rótulos: os intervalos associados aos atributos compõem o antecedente da regra e o grupo ao qual o rótulo está associado compõe o consequente. Quando o grupo possui rótulo com dois ou mais intervalos, o conectivo $e$ é utilizado. As regras a seguir exemplificam esta etapa para a BDM:

- R1: Se $A t t r_{1}=[3,1 \sim 5,3]$ então Grupo 1;

- R2: Se $A t t r_{1}=[5,8 \sim 7,8]$ então Grupo 2;

- R3: Se $A t t r_{1}=[8,5 \sim 9,8]$ e Attr $_{2}=[7,3 \sim 8,5]$ então Grupo 3.

Para classificação de um novo exemplo são avaliadas todas as regras, atribuindo-o ao grupo cuja regra obtiver o maior grau de ativação (GA). O GA é o valor resultante da pertinência de uma regra no processo de inferência. Caso haja mais de um componente no antecedente da regra, o GA será determinado por aquele que apresentar menor valor de pertinência.

Para simplificação, as faixas de cada atributo serão nomeadas faixa fi,j $_{j}$, em que $i$ representa o atributo e $j$ o grupo. Para o exemplo da BDM, a nomeação das faixas é apresentada na Tabela 3.

Supondo um novo exemplo com $A t t r_{1}=6,1$ e $A t t r_{2}=$ 7,4 , o modelo proposto irá calcular o grau de ativação para cada regra como mostra a seguir:
Tabela 3. Nomeações das faixas para BDM.

\begin{tabular}{|c|c|c|}
\hline Faixa & Representação & Valor \\
\hline faixa $_{1,1}$ & Faixa do $A t t r_{1}$ para Grupo 1 & {$[3,1 \sim 5,3]$} \\
\hline faixa $_{1,2}$ & Faixa do $A t t r_{1}$ para Grupo 2 & {$[5,8 \sim 7,8]$} \\
\hline faixa $_{1,3}$ & Faixa do $A t t r_{1}$ para Grupo 3 & {$[8,5 \sim 9,8]$} \\
\hline faixa $_{2,3}$ & Faixa do $A t t r_{2}$ para Grupo 3 & {$[7,3 \sim 8,5]$} \\
\hline
\end{tabular}

- $\mathrm{GA}(\mathrm{R} 1)=\min \left(\mu_{\text {aixa }_{1,1}}(6.1)\right)=0,46$;

- $\mathrm{GA}(\mathrm{R} 2)=\min \left(\mu_{\text {faixa }_{1,2}}(6.1)\right)=1$;

- $\mathrm{GA}(\mathrm{R} 3)=\min \left(\mu_{\text {faixa }_{1,3}}(6.1), \mu_{\text {faixa }_{2,1}}(7.4)\right)=\min (0,1)=$ 0 .

De acordo com o GA obtido, o novo elemento é associado ao grupo 2.

\subsection{Bases de Dados}

Nesta seção são apresentadas as bases de dados utilizadas nos testes do modelo proposto. São estas: Íris ${ }^{1}$, Sementes $^{2}$ e Doenças do Fígado ${ }^{3}$.

Íris A base Íris é formada por um conjunto de dados de três tipos diferentes da planta Íris: Setosa, Versicolor e Virginica. A base é composta por 50 elementos de cada tipo da planta. Os 150 elementos presentes nesta base são representados por 4 características: comprimento da sépala (CS), largura da sépala (LS), comprimento da pétala (CP) e largura da pétala (LP). Todos esses valores são expressos em centímetros.

Sementes Esta base refere-se à identificação de sementes de trigo e foi apresentada por Kulczycki e Charytanowicz Kulczycki and Charytanowicz (2011) . A base é composta por três diferentes tipos de trigo: Kama, Rosa e Canadian. Os 210 elementos presentes nesta base são representados por 7 características geométricas que formam o conjunto de entrada: área, perímetro, densidade, comprimento da semente (CS), largura da semente (LS), coeficiente de assimetria (CA) e comprimento do sulco da semente (CSS).

Doenças do Fígado - BUPA A base Doenças do Fígado foi criada pelo BUPA Medical Research, e contêm informações sobre exames de sangue consideradas sensíveis à problemas no fígado. A base possui informações de 345 pacientes do sexo masculino, representados por 5 características do exames e 1 referente ao número de bebidas alocólicas consumidas por dia, sendo essas: MCV, Alkphos, SGPT, SGOT, Gammagt e Drinks, respectivamente.

\subsection{Métricas de Avaliação}

A avaliação da eficiência do modelo de classificação proposto é realizada de duas formas: avaliando cada regra através das medidas baseada na matriz de contingência e avaliando o modelo de maneira geral por meio da acurácia.

No mundo real nem sempre é possível encontrar um modelo que resolva todo o problema, dessa forma se faz necessário uma avaliação das regras individualmente que podem resolver com precisão partes do problema. Entretanto, para comparação com demais modelos de

\footnotetext{
1 https://archive.ics.uci.edu/ml/datasets/iris

2 https://archive.ics.uci.edu/ml/datasets/seeds

3 https://archive.ics.uci.edu/ml/datasets/liver+disorders
} 
classificação, que não utilizam a formação de regras, as medidas de acurácia, porcentagem de acerto considerando todos os elementos, e o desvio padrão da acurácia podem ser utilizadas.

Segundo Freitas (1999) e Prati et al. (2002), a maioria das medidas de avaliação de regras estão baseadas na matriz de contingência, como exemplificada na Tabela 4. Considerando uma regra no formato Antecendente $\rightarrow$ Consequente, ou resumidamente $B \rightarrow H$, a matriz de contingência avalia as possíveis combinações de valores para os termos, representando por $B$ o conjunto de exemplos para os quais o antecedente da regra é verdadeiro e $\bar{B}$ seu complemento, denotando o conjunto de exemplos para os quais a condição da regra é falsa; analogamente ao consequente $H$ e $\bar{H}$.

Tabela 4. Matriz de Contingência para uma regra.

\begin{tabular}{l|l|l|l} 
& $H$ & $\bar{H}$ & \\
\hline$B$ & $h b$ & $\bar{h} b$ & $b$ \\
\hline $\bar{B}$ & $h \bar{b}$ & $\overline{h b}$ & $\bar{b}$ \\
\hline & $h$ & $\bar{h}$ & $n_{e x}$
\end{tabular}

onde:

- $h b=$ numero de elementos onde $H$ e $B$ são verdades;

- $h \bar{b}=$ numero de elementos onde $H$ é verdade e $B$ falso;

- $\bar{h} b=$ numero de elementos onde $H$ é falso e $B$ é verdade;

- $\overline{h b}=$ numero de elementos onde $H$ e $B$ são falsos;

- $b=$ numero de elementos onde $B$ é verdade;

- $\bar{b}=$ numero de elementos onde $B$ é falso;

- $h=$ numero de elementos onde $H$ é verdade;

- $\bar{h}=$ numero de elementos onde $H$ é falso;

- $n_{e x}=$ número total de elementos.

Utilizando a matriz de contingência é possível calcular medidas de avaliação para as regras (R), como: precisão, sensitividade e especificidade (Prati et al., 2002).

A precisão de uma regra $($ Pecisão $(R)=h b / b)$ é a medida do quanto esta regra é específica para o problema. A precisão pode ser definida como a probabilidade condicional de $H$ ser verdade, dado que $B$ é verdade. Quanto maior, mais precisamente a regra cobre a classe em questão.

A sensitividade (Sensitividade $(R)=h b / h$ ) é a medida do número relativo de exemplos da classe prevista em $H$ cobertos pela regra, ou seja, a probabilidade condicional de $B$ ser verdadeiro dado que $H$ é verdade. Quanto maior a sensitividade, mais exemplos são cobertos pela regra.

A especificidade $($ Especificidade $(R)=\overline{h b} / \bar{h})$ é o correspondente à completeza, porém para os exemplos que não são cobertos pela regra, ou seja, a probabilidade de $B$ ser falso dado que $H$ é falso.

\section{RESULTADOS}

O modelo proposto e os algoritmos utilizados para comparação, foram implementado utilizando a ferramenta $M A$ TLAB Math- Works ${ }^{4}$. Os classificadores escolhidos foram Multilayer Perceptron (MLP) e K Nearest Neighbor

\footnotetext{
4 https://www.mathworks.com/
}

(KNN) (Keller et al., 1985), amplamente difundidos na literatura. Nos testes, a MLP utilizada foi a padrão do $M A T L A B$ que possui 10 neurônios em uma única camada oculta. O método de treinamento utilizado foi o de retroprapagação de Levenberg-Marquardt (Hagan and Menhaj, 1994). Para o algoritmo KNN o valor de $k$ foi definido empiricamente como igual à 5 .

Na etapa de agrupamento, definiu-se como o número de grupos a quantidade de classes em cada base de dados. $\mathrm{Na}$ base Doenças do Fígado o número de grupos foi definido empiricamente como igual à 2, uma vez que esta não apresenta atributo classe.

A avaliação geral do modelo é realizada sobre o conjunto de teste formado por $15 \%$ dos elementos de cada grupo, devido ao número de elementos presentes nas bases. Estes elementos são retirados após o agrupamento e a rotulação é realizada com os elementos restantes. Para definição das classes padronizou-se para cada elemento o grupo ao qual este está associado. O conjunto de teste é utilizado após o treinamento do modelo para calcular acurácia e desvio padrão. Este tipo de avaliação é conhecido como método holdout (Gibaja and Ventura, 2015), em cada teste foram feitas 10 execuções considerando como resultado final a média destas.

\subsection{Base Íris}

Após o agrupamento da base de dados utilizando o algoritmo K-means com $k$ igual à 3 , o modelo de rotulação foi aplicado aos grupos, formandos os rótulos.

Após a rotulação são geradas as funções e regras para cada grupo com base nos intervalos dos rótulos. Os atributos pertencentes à pelo menos um rótulo tem suas funções de pertinência calculadas, como por exemplo, o atributo comprimento da pétala $(\mathrm{CP})$ cuja função de pertinência é apresentada na Figura 3.

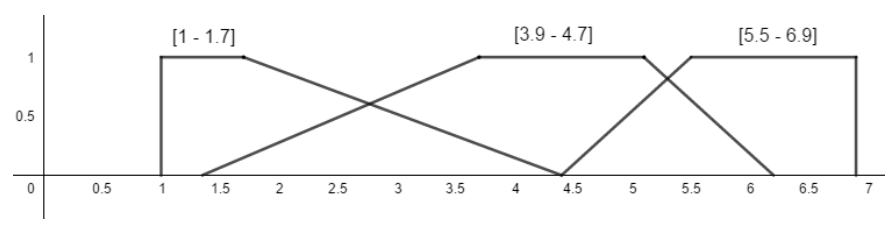

Figura 3. Função de pertinência para atributo CP.

O modelo proposto gera uma regra para cada grupo, como mostra a seguir:

- R1: Se CP $=[1 \sim 1,7]$ e $\mathrm{LP}=[0,1 \sim 0,4]$ então Grupo 1;

- R2: Se CS $=[6,3 \sim 7]$ e LS $=[2,8 \sim 3,2]$ e CP $=$ $[5,5 \sim 6,9]$ e $\mathrm{LP}=[1,9 \sim 2,5]$ então Grupo 2

- R3: Se CP $=[3,9 \sim 4,7]$ e $\mathrm{LP}=[1,1 \sim 1,5]$ então Grupo 3.

Tabela 5. Medidas de avaliação das regras para base Íris.

\begin{tabular}{|l|c|c|c|}
\hline & R1 & R2 & R3 \\
\hline Precisão (\%) & 100 & 100 & 100 \\
\hline Sensitividade (\%) & 90,48 & 21,21 & 48,08 \\
\hline Especificidade (\%) & 100 & 100 & 100 \\
\hline
\end{tabular}


Ao observar a Tabela 5 notam-se altas taxas de precisão e especificidade, isso reflete o fato dos rótulos que compõem as regras serem intrinsecamente ligados aos dados de cada grupo. A baixa taxa de sensitividade para a $R 2$ pode ser causada pela maior quantidade de componentes no antecedente da regra.

\subsection{Base Sementes}

O agrupamento da base de dados foi realizado pelo algoritmo $K$-means com $k$ igual à 3 . Com os grupos gerados, o modelo de rotulação é aplicado formando os rótulos. Após o processo de rotulação dos grupos são formados as funções e regras. Para exemplificar uma função de pertinência, a Figura 4 apresenta a função do atributo Área.

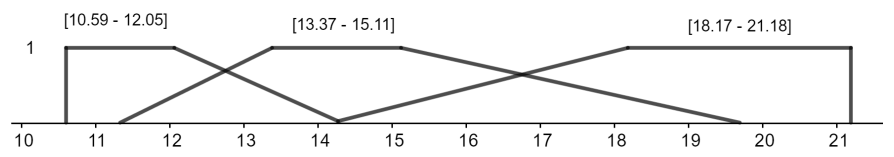

Figura 4. Função de pertinência do atributo Área.

Seguindo o modelo, as regras para cada grupo são formadas a partir dos rótulos, como pode ser observado a seguir.:

- R1: Se Área $=[10,59 \sim 12,05]$ e Perímetro $=[13,31$ $\sim$ 14,02] então Grupo 1;

- R2: Se Área $=[18,17 \sim 21,18]$ e $C S=[6,08 \sim 6,67]$ então Grupo 2;

- R3: Se Área $=[13,37 \sim 15,11]$ então Grupo 3 .

A partir das regras é possível cacular as medidas de avaliação obtidas da matriz de contingência. Na Tabela 6 podese observar os resultados obtidos.

Tabela 6. Medidas de avaliação das regras para base Sementes.

\begin{tabular}{|l|l|c|l|}
\hline & R1 & R2 & R3 \\
\hline Precisão (\%) & 94,87 & 100 & 97,14 \\
\hline Sensitividade (\%) & 58,73 & 63,49 & 53,57 \\
\hline Especificidade (\%) & 98,41 & 100 & 99,21 \\
\hline
\end{tabular}

\subsection{Doenças do Fígado - BUPA}

O agrupamento da base de dados utilizou o parâmetro $k$ igual à 2. Os rótulos dos grupos obtidos pelo modelo de rotulação são formadas as regras e funções. Como todos os rótulos são formados por apenas um atributo (Gammagt), é necessário somente uma função de pertinência para classificação de novos elementos, como mostra a regra a seguir:

- R1: Se Gammagt $=[5 \sim 27]$ então Grupo 1 ;

- R2: Se Gammagt $=[85 \sim 297]$ então Grupo 2.

Os resultados das medidas obtidas pela matriz de contigência das regras formadas para a base Doenças do Fígado são apresentados na Tabela 7 .

Novamente os resultados obtidos para as métricas demostram que as regras formadas a partir dos rótulos possuem altas taxas de precisão de especificidade.

\subsection{Avaliação Geral}

Na Tabela 8 são apresentados os resultados para as bases Iris, Seeds e BUPA considerando a medida de avaliação Acurácia (Acc) e Desvio Padrão (DP).

Obervando os resultados da Tabela 8 observa-se que o modelo proposto obteve uma taxa de acurácia menor que os algoritmos usados na comparação. Avalia-se que, uma vez que a formação das regras parte dos rótulos, estes possam afetar diretamente o desempenho do modelo proposto. Como os modelos de rotulação geram apenas um rótulo para cada grupo, consequentemente uma regra, estes podem limitar o desempenho do classificador.

\section{DISCUSSÃO}

Ao observar os resultados dos testes dois pontos podem ser discutidos em relação à acurácia obtida pelo modelo proposto em comparação aos demais classificadores. Como pode ser observado na Tabela 8, as médias das acurácias obtidas para base Íris foram relativamente próximas entre si, entretanto, nos testes realizados na base Sementes, o modelo proposto obteve uma acurácia 4,7\% abaixo do melhor colocado, MLP, apesar de obter um desvio padrão menor. Tais resultados podem ser atribuídos à diferença entre as relevâncias encontradas pelo modelo de rotulação para os atributos das duas bases de dados. Em Lopes et al. (2016) para base Sementes, a relevância dos atributos utilizados para formação dos rótulos variam em torno de 90\%, enquanto na base Íris a relevância dos atributos variam em torno de $95 \%$.

O segundo ponto importante está relacionado com a quantidade de rótulos representando um grupo, e consequentemente na quantidade de regras geradas. Uma maior quantidade de rótulos poderia representar o grupo com maior precisão, resultando em uma maior quantidade de regras e implicando em melhores resultados na classificação.

É importante destacar ainda que a classificação no modelo proposto é realizada após uma interpretação dos grupos de forma automática pelos modelos de rotulação, fornecendo além do classificador treinado, informações claras, concisas e independentes da subjetividade do especialista; contrapondo a abordagem dos demais algoritmos presentes na literatura para classificação logo após o agrupamento que podem não ser satisfatórios, visto que não se conhece o que cada grupo representa.

Tabela 7. Medidas de avaliação das regras para base Doenças do Fígado.

\begin{tabular}{|l|c|c|}
\hline & R1 & R2 \\
\hline Precisão (\%) & 100 & 100 \\
\hline Sensitividade (\%) & 62,2 & 87,1 \\
\hline Especificidade (\%) & 100 & 100 \\
\hline
\end{tabular}

Tabela 8. Resultados da acurácia para as bases Iris, Seeds e BUPA.

\begin{tabular}{|c|c|c|c|c|c|c|}
\cline { 2 - 7 } \multicolumn{1}{c|}{} & \multicolumn{2}{c|}{ MLP } & \multicolumn{2}{c|}{ KNN } & \multicolumn{2}{c|}{ Modelo } \\
\hline Base & Acc & DP & Acc & DP & Acc & DP \\
\hline Iris & $95,3 \%$ & 6,3 & $95,3 \%$ & 5,4 & $93,3 \%$ & 6,2 \\
\hline Seeds & $97,7 \%$ & 4,2 & $96,8 \%$ & 1,5 & $93 \%$ & 3 \\
\hline BUPA & $97,6 \%$ & 1,2 & $95,9 \%$ & 2,7 & $95,1 \%$ & 3,2 \\
\hline
\end{tabular}




\section{CONCLUSÃO}

Diversos trabalhos tem se dedicado à combinação de técnicas baseadas em aprendizagem de máquina supervisionada e não supervisionada. Em geral, estas técnicas estão estruturadas de modo à incluir entre elas uma etapa de análise dos grupos desempenhada por modelos matemáticos ou por um especialista. Neste trabalho foi apresentado um modelo de classificação que utiliza a rotulação como análise dos grupos, no qual os rótulos gerados formam as regras e funções de pertinência.

A avaliação do modelo foi realizada ao comparar à acurácia obtidas em três bases com outras técnicas de classificação (KNN e MLP). Além disso, as regras foram analisadas individualmente utilizando as medidas da matriz de contingência, e apresentaram alta porcentagem de precisão e especificidade obtendo $100 \%$ em todas as regras na base Íris e Doenças do Fígado. Para base Sementes, as taxas de precisão e especificidade mantiveram-se acima de 94,87 \%. O resultado da acurácia geral e desvio padrão, quando comparado com outros algoritmos, foram relativamente próximos, embora possam ser melhorados.

Como trabalhos futuros, propõe-se um mecanismo para atualização das regras, além de um estudo para o cálculo dos segmentos de retas na construção das funções de pertinências com o objetivo de melhor adequar as funções ao problema.

\section{AGRADECIMENTOS}

Agradecemos ao Programa de Pós-Graduação em Ciência da Computação da Universidade Federal do Piauí pelo suporte ao trabalho.

\section{REFERÊNCIAS}

Atilgan, C. and Nasibov, E. (2016). A memory efficient distributed fuzzy joint points clustering algorithm. In 2016 IEEE 10th International Conference on Application of Information and Communication Technologies (AICT), $1-5$. IEEE.

Bezdek, J.C. (1981). Objective function clustering. In Pattern recognition with fuzzy objective function algorithms, 43-93. Springer.

Biscarri, F., Monedero, I., García, A., Guerrero, J.I., and León, C. (2017). Electricity clustering framework for automatic classification of customer loads. Expert Systems with Applications, 86, 54-63.

Elyasigomari, V., Mirjafari, M., Screen, H.R., and Shaheed, M.H. (2015). Cancer classification using a novel gene selection approach by means of shuffling based on data clustering with optimization. Applied Soft Computing, 35, 43-51.

Ester, M., Kriegel, H.P., Sander, J., Xu, X., et al. (1996). A density-based algorithm for discovering clusters in large spatial databases with noise. In $K d d$, volume $96,226-$ 231.

Freitas, A.A. (1999). On rule interestingness measures. In Research and Development in Expert Systems XV, 147158. Springer.

Gan, H., Huang, R., Luo, Z., Xi, X., and Gao, Y. (2018). On using supervised clustering analysis to improve clas- sification performance. Information Sciences, 454, 216228.

Gibaja, E. and Ventura, S. (2015). A tutorial on multilabel learning. ACM Computing Surveys (CSUR), 47(3), 52.

Guha, S., Rastogi, R., and Shim, K. (1998). Cure: an efficient clustering algorithm for large databases. In ACM Sigmod Record, volume 27, 73-84. ACM.

Hagan, M.T. and Menhaj, M.B. (1994). Training feedforward networks with the marquardt algorithm. IEEE transactions on Neural Networks, 5(6), 989-993.

Jamshidi, M., Vadiee, N., and Ross, T. (1993). Fuzzy logic and control: software and hardware applications, volume 2. Pearson Education.

Keller, J.M., Gray, M.R., and Givens, J.A. (1985). A fuzzy k-nearest neighbor algorithm. IEEE transactions on systems, man, and cybernetics, (4), 580-585.

Kulczycki, P. and Charytanowicz, M. (2011). A complete gradient clustering algorithm. In International Conference on Artificial Intelligence and Computational Intelligence, 497-504. Springer.

Lopes, L.A., Machado, V.P., Rabêlo, R.A., Fernandes, R.A., and Lima, B.V. (2016). Automatic labelling of clusters of discrete and continuous data with supervised machine learning. Knowledge-Based Systems, 106, 231241.

Machado, V.P., Ribeiro Filho, V.P., and de Andrade Lira, R. (2015). Rotulação de grupos utilizando conjuntos fuzzy. In Simpósio Brasileiro de Automação Inteligente$S B A I, 2$.

MacQueen, J. et al. (1967). Some methods for classification and analysis of multivariate observations. In Proceedings of the fifth Berkeley symposium on mathematical statistics and probability, volume 1, 281-297. Oakland, CA, USA.

Milaré, C.R., de LF de Carvalho, A.C., and Monard, M.C. (2002). An approach to explain neural networks using symbolic algorithms. International Journal of Computational Intelligence and Applications, 2(04), 365-376.

Nomura, H., Hayashi, I., and Wakami, N. (1992). A learning method of fuzzy inference rules by descent method. In IEEE International Conference on Fuzzy Systems, 203-210. IEEE.

Pedrycz, W. and Gomide, F. (1998). An introduction to fuzzy sets: analysis and design. Mit Press.

Prati, R.C., Baranauskas, J.A., and Monard, M.C. (2002). Padronizaçao da sintaxe e informaçoes sobre regras induzidas a partir de algoritmos de aprendizado de máquina simbólico. Revista Eletrônica de Iniciaçao Cientrfica, 2(3), 21.

Russell, S.J. and Norvig, P. (2016). Artificial intelligence: a modern approach. Malaysia; Pearson Education Limited.

Safavian, S.R. and Landgrebe, D. (1991). A survey of decision tree classifier methodology. IEEE transactions on systems, man, and cybernetics, 21(3), 660-674.

Silva, I.d., Spatti, D.H., and Flauzino, R.A. (2010). Redes neurais artificiais para engenharia e ciências aplicadas. São Paulo: Artliber, 23(5), 33-111.

Zadeh, L.A. et al. (1965). Fuzzy sets. Information and control, 8(3), 338-353.

Zimmermann, H.J. (1996). Fuzzy control. In Fuzzy Set Theory - and Its Applications, 203-240. Springer. 